\title{
THE HAHN-BANACH THEOREM IMPLIES SINE'S MEAN ERGODIC THEOREM
}

\author{
RYOTARO SATO
}

\begin{abstract}
Using the Hahn-Banach theorem, a simple proof of Sine's mean ergodic theorem is given.
\end{abstract}

We shall prove the following.

TheOREM. Let $T$ be a linear contraction on a Banach space $B$. Then the ergodic averages $T_{n}=(1 / n)\left(I+T+\cdots+T^{n-1}\right)$ converge in the strong operator topology if and only if the fixed points of $T$ separate the fixed points of the adjoint operator $T^{*}$.

Proof. Letting $F$ be the fixed points of $T$ and $N$ the closed linear hull of the set $\{x-T x: x \in B\}$, the Hahn-Banach theorem implies that the second condition is equivalent to that $F+N$ is a dense subspace of $B$. And this condition immediately implies that for all $x \in B, T_{n} x$ converges in norm, as the norm of $B$ is complete. Conversely suppose $T_{n}$ converges strongly to $S$. Then $x=S x+(x-S x), T S x=S x$, and $T_{n}(x-S x)=T_{n} x-S x(n>1)$. Hence $S x \in F, \lim _{n} T_{n}(x-S x)=0$, and, for every invariant $x^{*}$ in $B^{*}$,

$$
\left\langle x-S x, x^{*}\right\rangle=\left\langle T_{n}(x-S x), x^{*}\right\rangle=0 .
$$

The Hahn-Banach theorem again implies that $x-S x \in N$. This shows that $B=F+N$, completing the proof.

In conclusion I should remark that the above argument applies in more general settings (cf. [1], [2], [3]).

ADDED IN PROOF. After the author submitted this paper, he learned from M. Lin that a similar argument appears in M. Lin, J. Montgomery and R. Sine, Change of velocity and ergodicity in flows and in Markov semi-groups, $Z$. Wahrscheinlichkeitstheorie und Verw. Gebiete 39 (1977), 197-211.

\section{BIBLIOGRAPHY}

1. S. P. Lloyd, On the mean ergodic theorem of Sine, Proc. Amer. Math. Soc. 56 (1976), 121-126.

2. R. J. Nagel, Mittelergodische Halbgnuppen linearer Operatoren, Ann. Inst. Fourier (Grenoble) (4) 23 (1973), 75-87.

3. R. Sato, On abstract mean ergodic theorems, Tôhoku Math. J. 30 (1978), 575-581.

4. R. Sine, A mean ergodic theorem, Proc. Amer. Math. Soc. 24 (1970), 438-439.

Department of Mathematics, Faculty of Science, Okayama University, Okayama 700, JAPAN

Received by the editors February 9, 1979.

AMS (MOS) subject classifications (1970). Primary 47A35.

Key words and phrases. Sine's mean ergodic theorem, Hahn-Banach theorem. 